\title{
Adaptive Assisting Effect of DAS for Elderly Drivers
}

\author{
Seunghee Hong ${ }^{1}$, Takafumi Asao ${ }^{2}$, Byungchan $\mathrm{Min}^{3}$, \\ Keisuke Suzuki ${ }^{1}$ and Shun'ichi Doi ${ }^{1}$ \\ ${ }^{1}$ Department of Intelligent Mechanical Systems Engineering s, \\ Kagawa University, tacamasctu, Japan \\ ${ }^{2}$ Department of Faculty of Engineering Science, Kansai University, Osaka, Japan \\ ${ }^{3}$ Department of Industrial Engineering and Management System, \\ Hanbat National University, Daejeon, Korea
}

\begin{abstract}
zeele22@naver.com
Abstract

A major cause of traffic accidents has been found in the human factors involved in driver's awareness during driving, and the effect of alarm was reported as the increase of awareness and the improving effect for property of perception, cognition and judgment of the driver. The arousal stimulus for driver by auditory-visual alert also affects to the physiological condition and the autonomic nervous system (ANS). Recent developments in multi near-infrared spectroscopy (MNIRS) have enabled the arousal level apparent with brain activity by measurement of hemoglobin concentrations as cerebral blood volume, as well as the ordinary biological signals like heart beats, finger plethysmogram and pulsation waves. In this study, the three kinds of auditory alert as pre-information, sound alert and voice guidance were used for adaptive assisting systems for the elderly driver and the influence of these alerts on the driver's psychosomatic state was examined using driving simulator for 30 minutes driving. Experiment was conducted with the subjects in a driving position as they were partially awakened by auditory-visual alert, repeatedly and measured their biological properties. As the results, after taking the effects of the adaptive assisting alert systems, the effect of arousal and awareness improvement was recognized.
\end{abstract}

Keywords: elderly driver, physiological response, driving assistance system, driving simulator

\section{Introduction}

Driving performance is influenced by age and driver's inner state. As shown in Fig. 1, three axes are supposed to express driving situations, which are driving situation, driving time, and driver axes. Lack or miss of any axis causes to lead to fatal traffic accidents. The driving performance has large differences among individuals because of driving skill due to age or career. Number of traffic accidents at intersections was increasing especially by elderly drivers. Therefore, Driver Assistance System (DAS) has been actively developed to support drivers. However, researchers had only mentioned improvement of driving behavior by DAS[1]; auditory and visual information were selected not to consider driver's arousal, irritability, and stress. The researchers should consider driver's inner state in addition to optimal timing or alert to support the drivers. Therefore, the researchers should make effort to identify driver's inner condition by their psycho-physiological reactions and physiological data [2][3].

There are some studies in which humans inner state's was estimated by subjective rating and measuring physiological signals. Increment of oxy-hemoglobin (below, Oxy[Hb]) and decrement of deoxy-hemoglobin (Deoxy[Hb].) reflect brain cortical activation. Multichannel near-infrared spectroscopy (MNIRS) was developed by Chance et al., which can successfully monitor human brain function [4][5]. Human physiological signal can be also estimated by using airpack pulse wave (APW) measured by air-pack sensors embedded in a driving seat[6]. The inner state of drivers had been estimated and analyzed by measuring galvanic skin response (GSR), electro-cardiogram (ECG), electroencephalogram (EEG), and skin temperature[7]. Min et al.[8] evaluated sensibility of driving speed and mode of vehicle for young passengers by autonomic responses and subjective assessment. The sympathetic nervous system of the passengers activated with increasing of the speed, when the passengers were tensed.

In this paper, we conducted two experiments to validate whether an optimal anti-stop assistance system was available or not for elderly drivers by using subjective assessments and physiological responses. We dealt with a driver support system by visual-audio alert 
that supports driver's cognition of environment as shown in Fig. 2. We examined effects of warning on driver's psychosomatic state by using a driving simulator (DS) and physiological indicators measured by MNIRS, ECG [8].

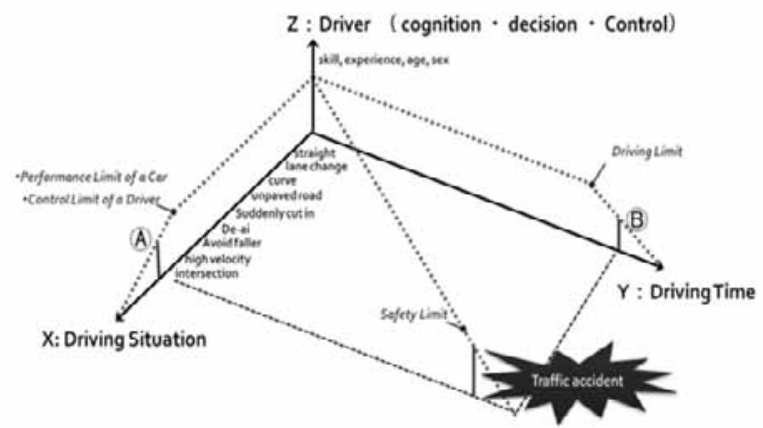

Fig. 1 Multidimensional Cause of Driving Accidents.

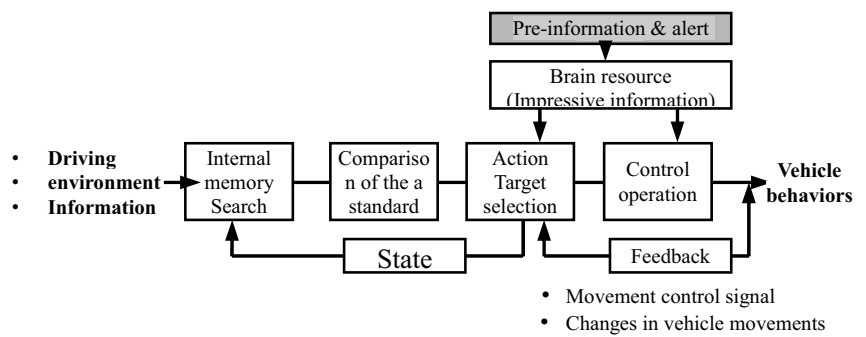

Fig. 2 Driver behavior model with pre-information \& alert system for driver

\section{Experimental Method}

\section{Subjects}

45 elderly drivers (67 to 79-years-old) who had been registered at an employment support center for elderly were enrolled for the pre-compliance testing. They are healthy people as possible to have a professional pre-recruited. And we demonstrated a test as a listening survey of personal information, a visual acuity, a color vision test, a useful field of view test (UFOV) and a Mini Mental State Examination (MMSE), in addition, performing Driving Style Questionnaire (DSQ), Workload Sensitivity Questionnaire (WSQ) [12], As the result, individual characteristics were categorized, and 10 people ( 8 males, 2 females) of them was chosen to agree with the experiment, but selected the data of 8 subjects as shown Table.1. Moreover, 10 young drivers (21 to 24 -yearsold) were also participated for comparison to elderly drivers. All the participants were informed consents for the experiments.

\section{Alerts Conditions and Procedure}

This experiment was executed for 30 minutes driving by using a driving simulator (DS-2000, Mitsubishi Precision, as shown in Fig. 3). Before the experiments, participants conducted practice driving for 3 minutes. The order of the alerts condition was counter-balancing for the subjects. The driving course was a two-lane straight. The subjects performed operation on a steering wheel, an accelerator and brake pedals with their usual driving postures. They had to keep a speed at $60 \mathrm{~km} / \mathrm{h}$, and stop short of an intersection.

We designed six alert conditions for a driving assistant alert system on a stop intersection by visual or auditory function as shown. For all conditions except for None condition, if the vehicle came close to the crossing, the system showed a stop sign on the LCD on the dashboard with "pong" sound, and after that, the system spoke "there is a stop intersection"; this alert is same as that for Info condition. Under Display condition, after alerts for the Info condition, an illustration was shown, that prompted the drivers to push the brake pedal. Under Low-freq beep condition, after the alerts for the Info condition, the system emitted beeps at $1.0 \mathrm{~Hz}$ for every $0.38 \mathrm{~s}$. Under High-freq beep condition, all alerts were same as those for the Low-freq beep condition, but the beep frequency and duration were $3.7 \mathrm{kHz}$ and $0.2 \mathrm{~s}$, respectively. Under Voice condition, after the alerts for Info condition, the system spoke "Brake smoothly" and after that, spoke "stop completely." Table 1 shows alert timing for each condition. All alert conditions were examined twice.

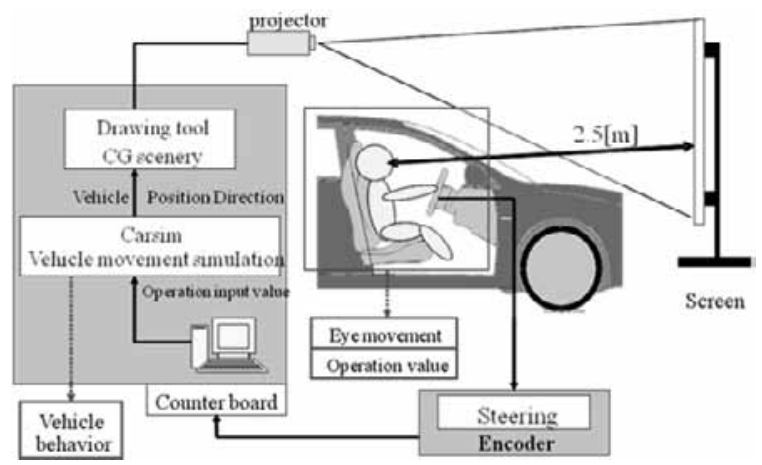

Fig. 3 Configuration of Driving Simulator.

Table. 1 Contents and Timing chart of the alert conditions

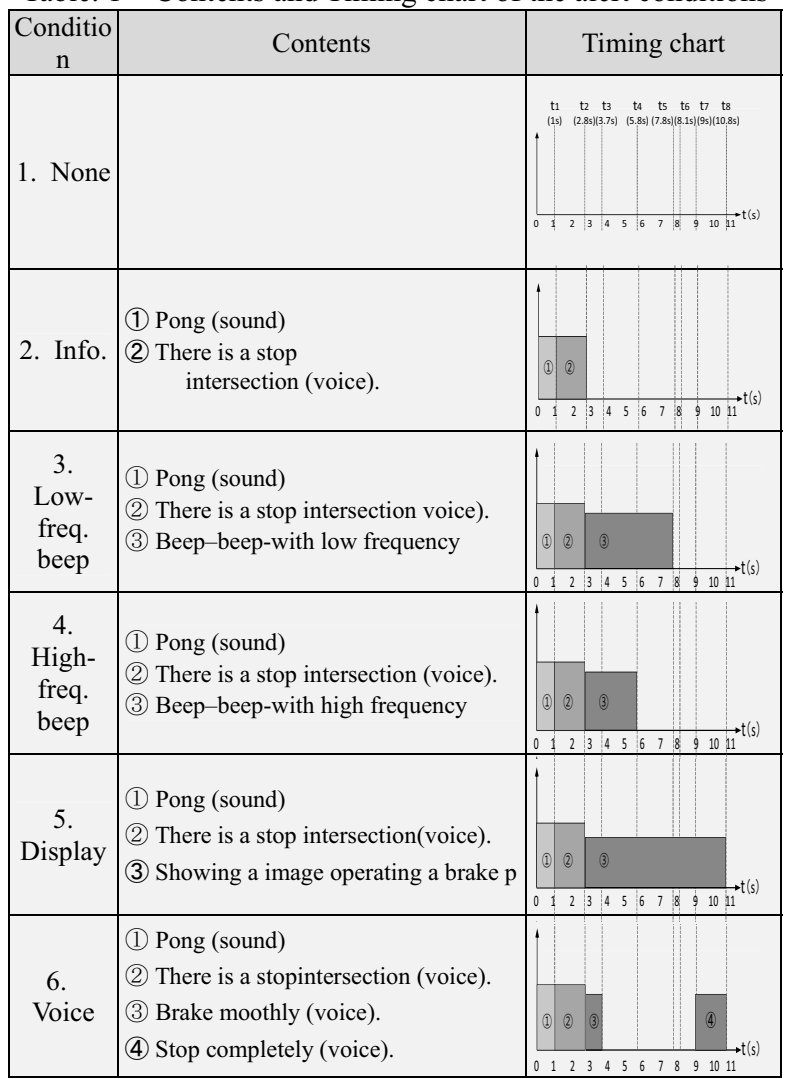


Bioinstrumentations and Physiological Indices

The multi-channel near-infrared spectroscopy (MNIRS, OEG-16, Spectratech.Ltd.) was used in order to measure brain blood flow at frontal region. In order to evaluate autonomic nervous system activities, electrocardiogram (ECG) was measured by using Power Lab (AD Instruments). The indices $\mathrm{HF}$ and $\mathrm{LF} / \mathrm{HFR}$ were calculated by the interval of heart beat (RRI), since RRI is influenced by autonomic nerves. When the value of $\mathrm{LF} / \mathrm{HF}$ becomes larger, human feels stress. The value of LF (low-frequency component) is influenced by sympathetic and parasympathetic nerve system. The value of $\mathrm{HF}$ (high-frequency component) is influenced by parasympathetic nerve. In this paper, $\mathrm{HF}$ and $\mathrm{LF} / \mathrm{HF}$ were used as indices of parasympathetic nerve and sympathetic nerve, respectively. These values were moving averaged for $180 \mathrm{~s}$ by sliding every $18 \mathrm{~s}$.

\section{Experimental Results}

\section{Classification of the older participants}

The participants conducted several previous tests, and the data was analyzed. Especially, we focused on a relation of between physical deterioration thought result of a useful field of view test (below, UFOV) or a Mini Mental State Examination (below, MMSE) and aging, besides affect it on driving performance under stop.

A correlation analysis of MMSE and UFOV score under aging was conducted. As the result, a significant correlation between aging and MMSE - UFOV score couldn't find. However, a significant correlation between MMSE and UFOV deterioration under aging and age do not always correspond with each other. chi square test ( $\chi^{2}$ test) was performed at the Excel program (ver. 2007). Results of MMSE had a normal distribution $[\mathrm{M}=28, \mathrm{Std}=1.981, \mathrm{MSE}=6.5233<$ critical value $=$ 9.488]. All of participants were over of 24 point, and even older people received 30 point was 15 persons. However, UFOV had no normal distribution.

Fig. 4 is a diagram for classification of all participants thought results of the MMSE and UFOV. The $\mathrm{x}$-axis of the diagram presented has to turn score of the UFOV into percentage, and the y-axis has to turn score of the MMSE into percentage. From the diagram, groups were separated into the group $\mathrm{O}_{-} \mathrm{A}$ in the top 25 th percentile, the group $\mathrm{B}$ from the 50 percentile to 74th percentile, the group $\mathrm{O}_{-} \mathrm{C}$ from the 25 percentile to 49th percentile and finally the group $\mathrm{O}_{-} \mathrm{D}$ from the 0th percentile to 24 th percentile. Received written consent from subject $22,23,25$ involved in the group O_A, subject 13, 32, 34 involved in the group B and subject 28,31 involved in the group O_D among all participants, the driving experiment with driving simulation has been conducted.

\section{The validity of each indicator}

Fig. 5 shows the results of HF and LF / HF obtained from the ECG, the concentration of hemoglobin obtained from the NIRS ch.2. The above graph was created based on the reports that have been reported in previous studies which suggested that the left side of the prefrontal cortex controls the activity of the sympathetic nervous system and the right side of the brain is very relevant to parasympathetic.

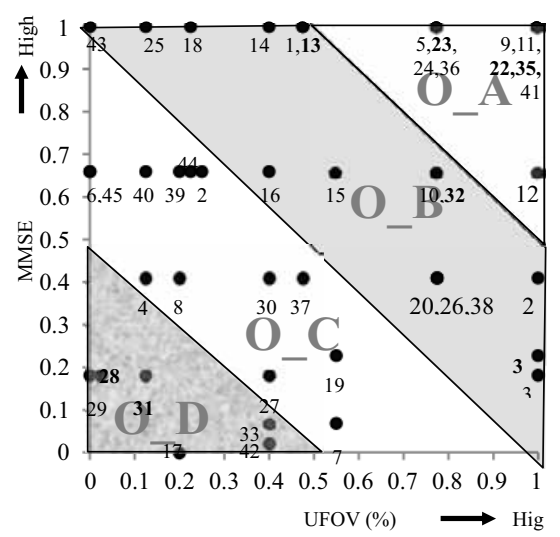

Fig.4 Distribution of all subjects by UFOV and MMSE

According to the results as a graph to create the following experimental results of the aged driver and the young drivers, it was confirmed that the concentration of the $\mathrm{Oxy}[\mathrm{Hb}]$ at channel. 2 that was adhering to the right prefrontal cortex and the LF / HF that shows the activity of sympathetic is very compatible. From the results, we got reasonable results in previous studies.

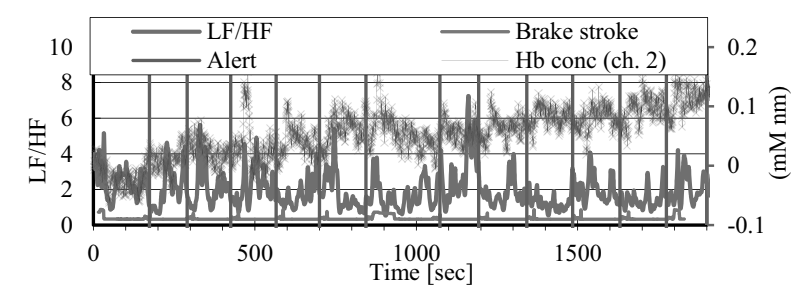

Fig. 5 Corresponding curve of LF/HF and Oxy[Hb]

Activation sympathetic of from $\mathrm{LH} / \mathrm{HF}$

Fig.6 shows the difference of LF / HF quantified between one condition and alert conditions. As a result, O_A group showed a significant increase in the info and the voice conditions. O_B group showed a significant increase in the info, the high.s and the voice conditions. There was a significant increase in the info, the low.s and the voice conditions at the O_D group. In addition, in the $\mathrm{Y}$ group, the degree of activity of a sympathetic is very low there was a significant increase in the high.s and the voice conditions.

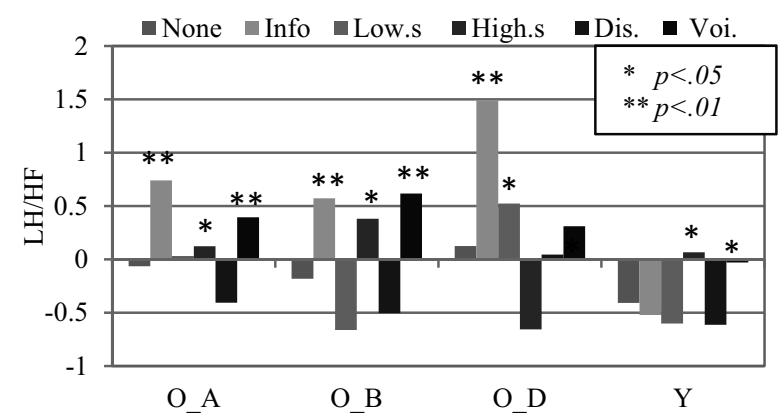

Fig. 6 Difference in LF/HF compared with the none condition. 


\section{Sympathetic activation of $\mathrm{Oxy}[\mathrm{Hb}]$}

After a hemoglobin concentration of oxygen in ch.2 normalized by $\mathrm{Z}$ score, $\mathrm{Oxy}[\mathrm{Hb}]$ was compared to that of the none condition as shown Fig7. As a result, although O_A group showed a very small increase, there was a significant increase in the high.s and voice conditions. O_B group has significantly increased in all conditions, compared to the none condition. As well as $\mathrm{O} \_\mathrm{B}$, in group $\mathrm{O} \_\mathrm{D}$ and $\mathrm{Y}$, there was a significant increase in all conditions.

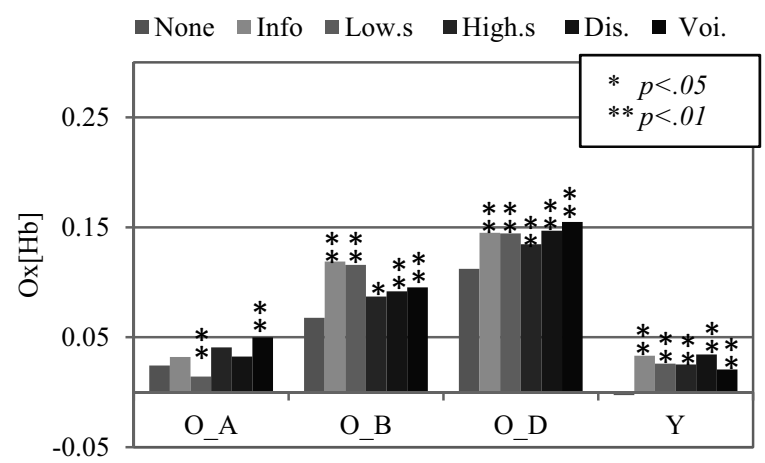

Fig. 7 Difference in Oxy[Hb] compared with the none condition.

\section{Discussion}

In this study, we tryed to get the biological reaction of older driver provided the alerts which induced the stop at the unsignalized intersection compared to the driver of college students. As an indicator of the biological reaction, HF (parasympathetic activity) and $\mathrm{LF} / \mathrm{HF}$ (the activity of the sympathetic nervous system) from ECG, oxygenated hemoglobin concentration obtained by NIRS were measured. The Table 3 shows the results of significant differences from physiological signals. As shown in the change in LF / HF of the following, significant activity of the sympathetic nerve is present in the info conditions of the entire elderly group. In the voice condition, we made sure that there was a significant effect on a group of the young and the elderly. Then, change in Hb.conc and found more responsive effects, compared with the LF / HF in the ECG. Oxy[Hb] in group O_A has increased in the high.s and voice conditions compared with the none condition. And in O_B, O_D and $\mathrm{Y}$ group, significant increase of $\mathrm{Oxy}[\mathrm{Hb}]$ have been discovered for all alert.

Table. 3 Change in LF/HF and Oxy[Hb] after alerts

\begin{tabular}{|c|c|c|c|c|c|c|c|c|c|c|}
\hline & \multicolumn{5}{|c|}{ Change in LF/HF } & \multicolumn{5}{|c|}{ Change in $\mathrm{Hb}$. conc ( $\mathrm{mM} \mathrm{nm})$} \\
\hline & $\begin{array}{c}\text { Inf } \\
0\end{array}$ & $\begin{array}{l}\text { Lo } \\
\text { w.s }\end{array}$ & $\begin{array}{l}\text { Hig } \\
\text { h.s }\end{array}$ & $\begin{array}{c}\text { Dis } \\
\text { pla } \\
\mathrm{y}\end{array}$ & $\begin{array}{l}\text { Voi } \\
\text { ce }\end{array}$ & Info & $\begin{array}{l}\text { Lo } \\
\text { w.s }\end{array}$ & $\begin{array}{l}\text { Hig } \\
\text { h.s }\end{array}$ & $\begin{array}{c}\text { Dis } \\
\text { pla } \\
\text { y }\end{array}$ & $\begin{array}{l}\text { Voi } \\
\text { ce }\end{array}$ \\
\hline O_A & $\begin{array}{c}* * \\
(0.000)\end{array}$ & $\begin{array}{c}- \\
(0.286)\end{array}$ & $\begin{array}{c}- \\
(0.153)\end{array}$ & $\begin{array}{c}- \\
(0.544)\end{array}$ & $\begin{array}{c}* * \\
(0.001)\end{array}$ & $(0.447)$ & $\overline{(0.139)}$ & $\begin{array}{c}* * \\
(0.101)\end{array}$ & $\begin{array}{c}- \\
(0.494)\end{array}$ & $\begin{array}{c}* * \\
(0.002)\end{array}$ \\
\hline O_B & $\begin{array}{c}* * \\
(0.000)\end{array}$ & $\begin{array}{c}- \\
(0.570)\end{array}$ & $\begin{array}{c}* \\
(0.023)\end{array}$ & $\begin{array}{c}- \\
(0.601)\end{array}$ & $\begin{array}{c}* * \\
(0.000)\end{array}$ & $\begin{array}{c}* * \\
(0.000)\end{array}$ & $\begin{array}{c}* * \\
(0.000)\end{array}$ & $\begin{array}{c}* * \\
(0.000)\end{array}$ & $\begin{array}{c}* * \\
(0.000)\end{array}$ & $\begin{array}{c}* * \\
(0.000)\end{array}$ \\
\hline O_D & $\begin{array}{c}* * \\
(0.000)\end{array}$ & $\begin{array}{c}* \\
(0.032)\end{array}$ & $\begin{array}{c}- \\
(0.478)\end{array}$ & $\begin{array}{c}- \\
(0.101)\end{array}$ & $\begin{array}{c}* \\
(0.040)\end{array}$ & $\begin{array}{c}* * \\
(0.000)\end{array}$ & $\begin{array}{c}* * \\
(0.000)\end{array}$ & $\begin{array}{c}* \\
(0.014)\end{array}$ & $\begin{array}{c}* * \\
(0.000)\end{array}$ & $\begin{array}{c}* * \\
(0.000)\end{array}$ \\
\hline $\mathrm{Y}$ & $\begin{array}{c}- \\
(0.897)\end{array}$ & $\begin{array}{c}- \\
(0.774)\end{array}$ & $\begin{array}{c}* \\
(0.012)\end{array}$ & $\begin{array}{c}- \\
(0.986)\end{array}$ & $\begin{array}{c}* \\
(0.019)\end{array}$ & $\begin{array}{c}* * \\
(0.000)\end{array}$ & $\begin{array}{c}* * \\
(0.000)\end{array}$ & $\begin{array}{c}* * \\
(0.000)\end{array}$ & $\begin{array}{c}* * \\
(0.000)\end{array}$ & $\begin{array}{c}* * \\
(0.000)\end{array}$ \\
\hline
\end{tabular}

\section{Conclusion}

In this study, the following results were revealed. From corresponding graph of blood flow in ch.2 by NIRS and result of LF / HF by ECG, local blood flow of right frontal cortex was confirmed to be associated with sympathetic component. From the results of the Difference in LF / HF before and after an alarm condition and without, the info conditions is also is very effective in the elderly, voice alarm and notification have found that not only the elderly, alarm effect is larger in young drivers. From sustained elevation of hemoglobin concentration of oxygen in the experimental time and a significant increase in cerebral blood flow of local instantaneous thought the difference in $\mathrm{Oxy}[\mathrm{Hb}]$ before and after an alarm condition and without, after the provision of alarm, an increase in the degree of concentration the effect of was discovered.

As the results, after the older people were divided into physical and cognitive abilities, we have verified the physiological effects of the alarm reaction. In future research, we plan to examine the differences in driving style according to each group of the elderly, to verify more deeply related to driving behavior.

\section{References}

[1] Gietelinkab, O., Ploega, J., Schutterb, B. D., Verhaegenb, M.: Development of advanced driver assistance systems with vehicle hardware-in-the-loop simulations; Vehicle System Dynamics, Vol. 44, No. 7, pp. 569-590 (2006).

[2] Hong, S., Harada, S., Onoue, K., Min, B., Suzuki, K., Doi, S.: Study of Adaptive Assisting Effect of Pre-Informative, Auditory-visual Alert for Elderly Drivers Using Near-Infrared Spectroscopy (NIRS); Proceedings of International Conference on Complex Medical Engineering, pp. 382-387 (2012).

[3] Hong, S., Onoue, K., Suzuki, K., Doi, S.: Study of Adaptive Assisting Effect of Auditory-visual Alert for Elderly Drivers using Physiological Signals; Proceedings of Annual Conference of Ergonomics Society of Korea, No. 1230951, pp. 156-166 (2012).

[4] Chance, B., Zhuang, Z., Unah, C., Alter, C., Lipton, L.: Cognition-activated low-frequency modulation of light; Neurobiology, Vol. 90, No. 8, pp. 3770-3774 (1993).

[5] Maki, A., Yamashita, Y., Ito, Y., Watanabe, E., Mayanagi, Y., Koizumi, H.: Spatial and temporal analysis of human motor activity using noninvasive NIR topography; Medical Physics, Vol. 22, No. 12, pp. 1997-2005 (1995).

[6] Fujita, E., Ogura, Y., Ochiai, N., Yasuda, E., et al: Development of simplified appraisal method of fatigue on sitting by finger plethysmogram; Journal of Japan Ergonomics Society, Vol. 40, No. 5, pp. 254-263 (2004).

[7] Hong, S., Min, B., Doi, S.: Study on Driving Performance of Aged Drivers at the Intersections Compared with Young Drivers; Proceedings of International Conference on Human-Computer Interaction, Lecture Notes in Computer Science, Vol. 6781, pp. 156-166 (2011).

[8] Min, B.C., Chung, S.C., Park, S.J., Kim, C.J., Sim, M.K., Sakamoto, K.: Autonomic responses of young passengers contingent to the speed and driving mode of a vehicle; International Journal of Industrial Ergonomics, Vol. 29, No. 4, pp. 187-198 (2002)

[9] Yoon, B.W., Morillo, C.A Cechetto D., Hachinski V.: Cerebral hemispheric lateralization in cardiac autonomic control. Archives of Neurology, Vol. 54, pp. 741-744 (1997) 